\title{
Biology and the art of abstraction
}

\author{
Petra Schwille $^{1}$ (D)
}

Received: 4 July 2017 / Accepted: 4 July 2017 /Published online: 29 July 2017

(C) International Union for Pure and Applied Biophysics (IUPAB) and Springer-Verlag GmbH Germany 2017

\section{Physicists in biology}

Despite the large number of trained physicists who are currently working in the life science fields, very few people outside academic research associate biology with a physicist's obvious sphere of action. In school educational curricula, physics usually makes its statements by simplifying phenomena to the extreme, mostly in terms of mass points and perfect oscillators. Biology, on the other hand, notoriously confronts the student with an ever-growing number of facts, which only the most diligent and selfmotivated students are happy, or even willing, to memorize. The result is - at least in Germany, but likely also in other Western countries - an almost complementary sorting of high school students with respect to their basic science education. This discrepancy more or less continues into university, and although most life science faculties have realized that a strong quantitative training curriculum is also essential for biologists and biochemists, mathematics and physics courses usually represent the "necessary evil" for many aspiring life scientists, not to mention medical doctors. On the other hand, although many universities nowadays offer academic courses in biophysics at the Master or even Bachelor level, biology is not an obvious field of specialization for most physics students, in contrast to mathematics, computer sciences, engineering, and chemistry.

This article is part of a Special Issue on 'IUPAB Edinburgh Congress' edited by Damien Hall.

Petra Schwille

schwille@biochem.mpg.de

1 Max Planck Institute of Biochemistry, Am Klopferspitz 18, 82152 Martinsried, Germany

\section{Impact of physical methods and technology}

Taking a closer look into the reality of just how and where physicists and biologists work side by side in the laboratory, the most fruitful collaborations are usually realized through the application of sophisticated technology, as developed by physicists and engineers, to emulate biological problems. At the present time, the largest impact on biosciences by physicsbased technology is likely the development of ultra-high resolution microscopy, such as cryo-electron tomography and fluorescence nanoscopy (Asano et al. 2016; Hell et al. 2015), while advances in computational routines for simulations and large data handling have resulted in great progress in all kinds of -omics approaches (Cox and Mann 2011). In these collaborations, the biology partners very often set the stage for defining the proper scientific question, while the physics partners are left with the role of being an assistant — albeit a highly sophisticated one - to reach the goals with the highest possible degree of quantitative rigour.

A second but much smaller class of very successful interdisciplinary collaborations is that of theory-based physical modelling of particular biological phenomena, where existing physical theories, mostly from the fluid dynamics and the soft matter fields, are expanded and adapted to the very special aspects of living matter (Prost et al. 2015). These approaches very often focus on biological membranes or cytoskeletal polymers. In this respect, the recognition of the polymeric nature of biological molecules, and in particular the ability of these polymers to phase separately and by this behavior constitute heterogeneous environments, is currently among the most recognized and welcomed physical inspirations to the biosciences (Hyman et al. 2014). Even more fundamental, and reaching further into the inner workings of living systems, are theories of self-assembly and particularly of spatiotemporal self-organization (Turing 1952; Karsenti 2008), as well as 
network models (Alon 2007). These catalyse state-of-the-art combinations of theory and fluorescence or atomic force microscopy video-rate microscopy to quantitatively assess biological pattern formation and morphogenesis on the one hand and gene expression on the other, as well as their dependency on and robustness against stochastic fluctuations.

\section{The dogma of physiological relevance}

Despite several outstanding examples of conceptual crossovers between physics and biology having been published in recent years, representative publications in high-visibility journals with a wide, global audience of biologists is still relatively rare. The reason for this absence is best described by the magical term of "physiological relevance", being the key selective criterion applied by many editors and reviewers to distinguish research that life science researchers should be aware of from research that may only appeal to a limited audience with a particularly exquisite quantitative taste. It is only upon being confronted with the verdict of missing physiological relevance that physicists working in the life sciences realize that despite close collaborations with biologists, there is still a large fundamental divide between the two disciplinespecific scientific rationales.

\section{Pros and cons of abstraction}

The holy grail of the physicist is the derivation of phenomena from first principles, with ideally only a few variables, which should then be subject to measurement. With respect to designing experiments, this often results in reductionism, i.e., abstracting phenomena to minimal systems with as few parameters and observables as possible (Fig. 1). The art of abstraction is without doubt one of the most valued accomplishments of mankind; however, it appears to have astonishingly little attractiveness to modern biologists. In fact, abstraction even seems to be a relatively dubious effort for many of them, as far as living systems are concerned. Biologists often search for the truth in the details, and the discovery of exceptions from any possible rule seems to be much more tangible than the formulation of the rule in the first place. Whenever a cellular process has indeed been described to support a suggested model, this may be due to not having considered an important parameter, or molecule, supposed to complicate the model further. Almost every author of a biology-related study has at least once been confronted with the verdict of the (mostly, third) reviewer that a study is useless until it has verified the existing or missing influence of the reviewer's favourite protein or been carried out in another model organism. In other words, physiological relevance seems to be the conceptual opposite to abstraction, at least in its implications to the design of scientific studies.

\section{In biology, experience counts}

This apparent lack of interest in abstraction is certainly not due to a somehow begrudging character of biologists, but rather to their long-time experience with experiments on living systems, the results of which are only very rarely as unequivocal as physicists like to have them. And this again can be explained by the immense complexity of cells and organisms that has been acquired in more than three billions of years of evolution on earth. To generalize or abstract in a system of such enormous complexity can only be accomplished by zooming out considerably, or by focussing on a very specific aspect of limited connectivity (and consequently reduced relevance). Nowadays, the more appropriate approach for most

Fig. 1 How can biology be abstracted? There is a large conceptual divide between scalefree networks, as provided by modern proteomics (left, provided by Jan Rieckmann, Max Planck Institute of Biochemistry, Martinsried) and the minimal physical model of a cell as a spherical reaction compartment (right)
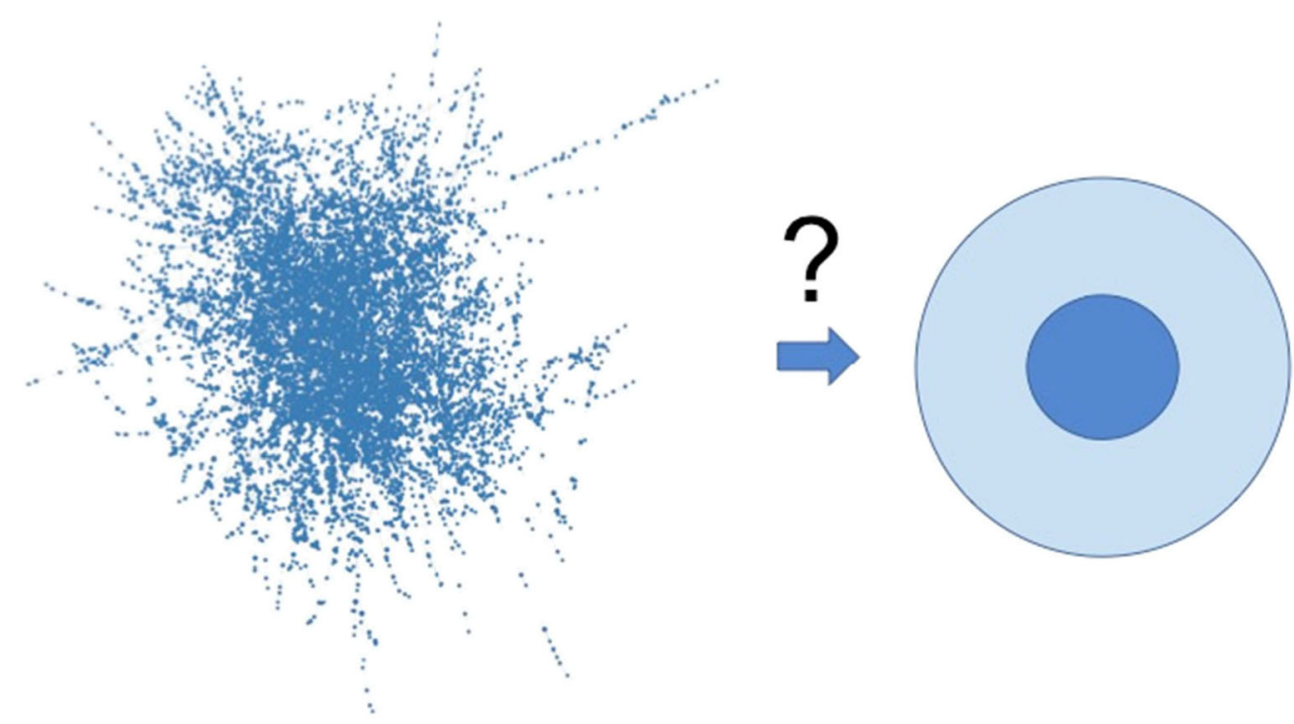
researchers concerned with a predictive and quantitative understanding of living systems is the systems level approach, where complex algorithms and large computing power compensate for a limited ability to handle close to a infinite number of - all relevant- parameters.

\section{The value of fundamental questions}

The question therefore is whether abstraction - given these different backgrounds - is generally useless in biology. By no means. In fact, I would like to advocate a renaissance of abstraction-based approaches, made possible by a greater emancipation of physicists working in the life sciences from particular goals mainly set by their biological collaborators that currently appear to be the most fashionable. In other words, I would like to encourage biophysicists to formulate their own fundamental questions to biology, which may or may not overlap to a significant extent with the questions that biologists consider most burning. Very exciting advances in this direction have been made in recent years by the recognition that relatively simple physical phenomena lie at the basis of many highly regulated, and thus extremely complex, cellular processes (Loose et al. 2008). The above-mentioned fluid phase separation (Hyman et al. 2014) that apparently constitutes the makeup of a number of essential organelles is only the most recent example. Most modern proteins indeed confer highly specialized catalytic activity, but this should not fool us into thinking that without them the particular biological process which they are involved in could not exist. Instead, it makes much more sense to assume that nearly every process that is now so highly regulated and finely tuned in a cell or organism may also exist in a much more rudimentary form, which would however be highly inefficient. Nevertheless, this abstraction-based avenue towards understanding biology from first principles is only in its infancy, although it could become a field of research attracting many more fellow physicists in the future into the life sciences and-importantly-not as auxiliary personnel developing technology, but as alternative drivers of the field.

Naturally, this also reflects on a different approach towards designing biological studies. Simply abstracting the models while still performing experiments in a highly complex environment such as the living cell or organism is doomed to remain an unsatisfactory strategy. Instead, the functional reconstitution of interesting phenomena with purified components into cell-free systems will become ever more relevant (Schwille 2011), despite the hardships posed by complicated biochemistry. It is exciting to witness that this approach of a bottom-up assembly of biological systems with defined functional modules is constantly growing in the biophysical community throughout Europe. Nothing is so complex that it renders abstraction unnecessary or useless - not even life.

\section{Compliance with ethical standards}

Conflict of interest Petra Schwille declares that she has no conflicts of interest.

Ethical approval This article does not contain any studies with human participants or animals performed by any of the authors.

\section{References}

Alon U (2007) Network motifs: theory and experimental approaches. Nat Rev Genet 8:450-461

Asano S, Engel BD, Baumeister W (2016) In situ cryo-electron tomography: a post-reductionist approach to structural biology. JMB 428: 332-343

Cox J, Mann M (2011) Quantitative, high-resolution proteomics for datadriven systems biology. Annu Rev Biochem 80:273-299

Hell SW, Sahl SJ, Bates M. et al. (2015) The 2015 super-resolution microscopy roadmap. J Phys D Appl Phys 48:443001

Hyman AA, Weber CA \& Jülicher F (2014) Liquid-liquid phase separation in biology. Ann Rev Cell Dev Biol 30:39-58

Karsenti E (2008) Self-organization in cell biology: a brief history. Nat Rev Mol Cell Biol 9:255-262

Loose M, Fischer-Friedrich E, Ries J, Kruse K, Schwille P (2008) Spatial regulators for bacterial cell division self-organize into surface waves in vitro. Science 320:789-792

Prost J, Juelicher F, Joanny J-F (2015) Active gel physics. Nat Phys 11: $111-117$

Schwille P (2011) Bottom-up synthetic biology: engineering in a tinkerer's world. Science 333:1252-1254

Turing A (1952) The Chemical Basis of Morphogenesis. Philos Trans R Soc Lond B 237:37-72 